\section{Liquid Biopsy for Predicting Bacillus Calmette-Guérin Unresponsiveness in Non-muscle-invasive Bladder Cancer}

Intravesical immunotherapy with bacillus Calmette-Guérin (BCG) is the gold-standard treatment for non-muscleinvasive bladder cancer (NMIBC) with a high risk of recurrence or progression. Unfortunately, despite treatment, $25-45 \%$ of patients will not benefit from therapy, and an additional $40 \%$ will ultimately require more aggressive treatment [1]. BCG-induced overexpression of PD-L1 has been described as one mechanism of acquired resistance to BCG in patients with NMIBC, and the US Food and Drug Administration (FDA) recently approved an anti-PD-1 agent as monotherapy in patients with BCG-unresponsive NMIBC [2]. Although baseline PD-L1 expression in tumor tissue predicts an unfavorable response to BCG [3], spatial and temporal tumor heterogeneity are major biological issues to overcome when using tumor tissues for predictive biomarker analysis, since PD-L1 might be inadequately represented in the biopsy specimen. To overcome these limitations, liquid biopsy for assessment of PD-L1 was identified as suitable for guiding therapeutic choice for patients who were candidates for PD-L1 inhibitors [4]. A role for enumeration of circulating tumor cells (CTCs) in improving current risk stratification models in high-risk NMIBC was recently proved in a study demonstrating that CTCs are detectable in almost $20 \%$ of NMIBC cases, who represent a subgroup of "super-high risk" patients requiring closer monitoring for local recurrence and/or progression of disease [5].

Here we explored PD-L1 expression in CTCs isolated from 20 selected CTC-positive patients (CTC cutoff $\geq 1$ ) prospectively enrolled before starting adjuvant BCG therapy for NMIBC. PD-L1 analysis was not performed for tumor tissues because of the scarcity of tissue sample available. Two different methods for CTC isolation were compared: the antigen-dependent FDA-approved CellSearch system and a size-based CTC isolation method (ScreenCell). A blood sample from a healthy donor and spiked SKBR3 cells were used as negative and positive controls, respectively. Patients were followed for median follow-up of 18 mo (range 3-20).

Table 1 - Clinicopathological features of the study cohort.

\begin{tabular}{|c|c|c|c|c|c|c|c|c|c|c|c|}
\hline \multirow[t]{3}{*}{ Pt } & \multirow{3}{*}{$\begin{array}{l}\text { Age } \\
(y r)\end{array}$} & \multirow[t]{3}{*}{ Gender } & \multicolumn{2}{|c|}{ Tumor } & \multirow[t]{3}{*}{ CIS } & \multicolumn{4}{|c|}{ CTC number } & \multirow{3}{*}{$\begin{array}{l}\text { TTRP } \\
\text { (mo) }\end{array}$} & \multirow[t]{3}{*}{ BCG response } \\
\hline & & & Stage & Grade & & \multicolumn{2}{|c|}{ CellSearch } & \multicolumn{2}{|c|}{ ScreenCell } & & \\
\hline & & & & & & Total & PD-L1 ${ }^{+}$ & Total & PD-L1 ${ }^{+}$ & & \\
\hline 1 & 77 & $\mathrm{M}$ & $\mathrm{T} 1$ & G3 & Yes & 3 & 3 & 4 & 4 & 6 & Unresponsive \\
\hline 2 & 65 & M & $\mathrm{T} 1$ & G3 & No & 4 & 4 & 5 & 4 & 8 & Unresponsive \\
\hline 3 & 71 & $\mathrm{M}$ & $\mathrm{T} 1$ & G3 & No & 3 & 3 & 4 & 3 & 5 & Unresponsive \\
\hline 4 & 59 & $\mathrm{~F}$ & $\mathrm{~T} 1$ & G3 & Yes & 2 & 2 & 3 & 3 & 3 & Unresponsive \\
\hline 5 & 70 & $\mathrm{M}$ & $\mathrm{T} 1$ & G3 & No & 2 & 2 & 3 & 2 & 12 & Unresponsive \\
\hline 6 & 64 & $\mathrm{~F}$ & $\mathrm{~T} 1$ & G3 & No & 2 & 2 & 3 & 2 & 9 & Unresponsive \\
\hline 7 & 56 & $\mathrm{~F}$ & $\mathrm{~T} 1$ & G3 & No & 2 & 1 & 5 & 2 & 12 & Unresponsive \\
\hline 8 & 66 & $\mathrm{M}$ & $\mathrm{T} 1$ & G3 & Yes & 3 & 3 & 4 & 3 & 8 & Unresponsive \\
\hline 9 & 79 & $\mathrm{M}$ & $\mathrm{T} 1$ & G3 & No & 0 & 0 & 0 & 0 & 15 & Unresponsive \\
\hline 10 & 75 & M & $\mathrm{T} 1$ & G3 & Yes & 1 & 1 & 2 & 2 & 9 & Unresponsive \\
\hline 11 & 71 & M & $\mathrm{T} 1$ & G3 & No & 2 & 2 & 4 & 2 & 3 & Unresponsive \\
\hline 12 & 52 & $\mathrm{~F}$ & $\mathrm{~T} 1$ & G3 & No & 2 & 2 & 4 & 4 & 6 & Unresponsive \\
\hline 13 & 66 & $\mathrm{M}$ & $\mathrm{T} 1$ & G3 & No & 0 & 0 & 0 & 0 & NA & Responsive \\
\hline 14 & 68 & M & $\mathrm{T} 1$ & G3 & Yes & 0 & 0 & 0 & 0 & NA & Responsive \\
\hline 15 & 70 & $\mathrm{M}$ & $\mathrm{T} 1$ & G3 & No & 0 & 0 & 0 & 0 & NA & Responsive \\
\hline 16 & 64 & M & $\mathrm{T} 1$ & G3 & No & 0 & 0 & 0 & 0 & NA & Responsive \\
\hline 17 & 59 & $\mathrm{M}$ & $\mathrm{T} 1$ & G3 & No & 0 & 0 & 0 & 0 & NA & Responsive \\
\hline 18 & 77 & $\mathrm{~F}$ & $\mathrm{~T} 1$ & G3 & No & 0 & 0 & 0 & 0 & NA & Responsive \\
\hline 19 & 71 & M & $\mathrm{T} 1$ & G3 & No & 0 & 0 & 0 & 0 & NA & Responsive \\
\hline 20 & 60 & $\mathrm{~F}$ & $\mathrm{~T} 1$ & G3 & No & 0 & 0 & 0 & 0 & NA & Responsive \\
\hline
\end{tabular}


Patients with recurrence or progression during therapy were included in a BCG-unresponsive group. During followup, no recurrence or tumor progression was observed in eight patients (40\%), while 12 patients were defined as BCGunresponsive (60\%). Among the latter, eight patients (66.7\%) had local recurrence and four (33.3\%) experienced progression to muscle-invasive disease. PD-L1-positive CTCs were found in 11/12 of the BCG-unresponsive group (92\%), while CTCs from the eight BCG-responsive patients stained negative for PD-L1, with a concordance rate of $100 \%$ between the two assays (Table 1). The slight difference in CTC number detected by the two assays might be explained by the low ability of CellSearch to detect CTCs in partial epithelial-mesenchymal transition.

This proof-of-concept study demonstrates for the first time the feasibility of a liquid biopsy test for PD-L1 assessment in high-risk NMIBC using two different assays. Although the standardized pre-analytical preparation of CTCs and inclusion of a positive control render CellSearch more suitable for clinical practice, ScreenCell is an easy technique with the advantage that it does not require complex instruments or adapted staff training. We suggest that baseline and serial assessment of PD-L1 using liquid biopsies might be a suitable tool for guiding therapeutic choice for patients who are candidates for PD-L1 inhibitors after BCG failure.

Conflicts of interest: The authors have nothing to disclose.

\section{References}

[1] Tse J, Singla N, Ghandour R, et al. Current advances in BCG-unresponsive non-muscle invasive bladder cancer. Expert Opin Investig Drugs 2019;9:757-70.
[2] Grimm MO, Bex A, De Santis M, et al. Safe use of immune checkpoint inhibitors in the multidisciplinary management of urological cancer: the European Association of Urology position in 2019. Eur Urol 2019;76:368-80.

[3] Kates M, Matoso A, Choi W, et al. Adaptive immune resistance to intravesical BCG in non-muscle invasive bladder cancer: implications for prospective BCG-unresponsive trials. Clin Cancer Res 2020;26:882-91.

[4] Nicolazzo C, Raimondi C, Mancini M, et al. Monitoring PD-L1 positive circulating tumor cells in non-small cell lung cancer patients treated with the PD-1 inhibitor nivolumab. Sci Rep 2016;6:31726. http://dx.doi.org/10.1038/srep31726.

[5] Nicolazzo C, Busetto GM, Gradilone A, et al. Circulating tumor cells identify patients with super-high-risk non-muscle-invasive bladder cancer: updated outcome analysis of a prospective single-center trial. Oncologist 2019;24:612-6.

Chiara Nicolazzo ${ }^{\mathrm{a}}$ Ettore de Berardinis ${ }^{\mathrm{b}}$ Paola Gazzaniga ${ }^{\mathrm{a}, *}$

${ }^{\mathrm{a} D e p a r t m e n t}$ of Molecular Medicine, Sapienza University of Rome, Rome, Italy

${ }^{\mathrm{b}}$ Department of Maternal-Child and Urological Sciences, Sapienza University of Rome, Rome, Italy

${ }^{*}$ Corresponding author. Department of Molecular Medicine, Sapienza University of Rome, Rome, Italy. E-mail address: paola.gazzaniga@uniroma1.it (P. Gazzaniga). Associate Editor: Ashish Kamat

July 22, 2020 